\title{
PROPUESTA CURRICULAR PARA LA IMPLEMENTACIÓN DE LA CARRERA DE INGENIERÍA DE SOFTWARE DE LA FUNDACIÓN UNIVERSITARIA INPAHU
}

\author{
Patricia Posso R.1 ${ }^{1}$ Heiver Cuesta-Dávila², Luis Alexis Plazas-Gómez ${ }^{3}$ \\ ${ }^{1}$ Docente investigadora, Maestría en Educación. Universidad Cooperativa de Colombia, sede Bogotá \\ Correo electrónico: patricia.posso@campusucc.educ.co \\ ${ }^{2}$ Coordinador de prácticas, Facultad de Ingeniería y Tecnologías de la Información, Fundación Universitaria Inpahu, Colombia \\ ${ }_{3}^{3}$ Docente de cátedra, Facultad de Ingenierías, Fundación Universitaria Los Libertadores, Colombia
}

Recibido: 10 de febrero del 2013. Aprobado: 25 de abril del 2013.

Cómo citar este artículo: P. Posso R., H. Cuesta-Dávila y L. A. Plazas-Gómez, "Propuesta curricular para la implementación de la carrera de Ingeniería de Software de la Fundación Universitaria Inpahu". Ingeniería Solidaria, Vol. 9, №. 16, pp. 141-151, Dic., 2013.

Resumen. Se presenta el resultado de la investigación "Reestructuración malla curricular para la carrera de ingeniería de software en Inpahu" (2011), que tiene como objetivo plantear las nuevas proyecciones del ingeniero de software, con base en el desarrollo de las competencias lógicas, creativas y operativas, unidas a las intelectuales, técnicas y tecnológicas. Para tal fin se plantea la implementación del modelo tricerebral de De Gregori [1] que plantea un cerebro de estructura triádica. Si el aprendizaje es un proceso de adaptación al medio en constante transformación y los estilos de aprendizaje son, entre otras cosas, la manera característica que los estudiantes tienen para adaptarse y vivir en el entorno, entonces, la forma como utilicen sus funciones cerebrales definirá su estilo de pensamiento y aprendizaje. La investigación pretende aportar a los estudiantes de Ingeniería de Software el desarrollo de su potencial cerebral encaminado hacia una sólida formación integral que posibilite su acertada inserción en la vida laboral.

Palabras clave: cerebro tríadico, competencias lógicas, creativas y operativas, currículo, ingeniería de software.

\section{Curriculum Proposal for a Degree in Software ENGINEERING AT THE FUNDACión Universitaria INPAHU}

Abstract. This paper presents the results of the research study titled "Restructuring the curriculum for a software engineering degree at Inpahu University" (2011), which presented an education plan based on development of logical, creative and operational skills in tandem with intellectual, technical and technological abilities. Accordingly, it was proposed that this plan incorporate the three-brain model of De Gregori [1] which posits a triadic brain structure. If learning is the process of adapting to a constantly changing environment and learning styles are, among other things, the typical way for students to adapt to and live in their environment, the way they use their brain functions will define their style of thinking and learning. The research seeks to help software engineering students develop their brain potential in moving towards a solid and holistic education that will facilitate a successful transition into working life.

Keywords: triadic brain, logical, creative, and operational skills, curriculum, software engineering.

\section{Proposta CUrRicular para A implementaÇão Do CURSO de ENGENHARIA DE SOFTWARE dA FUNDAÇÃo UNIVERSITÁRIA INPAHU}

Resumo. Neste artigo, apresenta-se o resultado da pesquisa "Reestruturação do currículo para o curso de engenharia de software em Inpahu" (2011), que tem como objetivo propor novas projeções do engenheiro de software, com base no desenvolvimento das competências lógicas, criativas e operativas, unidas às intelectuais, técnicas e tecnológicas. Para isso, propõe-se a implantação do modelo tricerebral de De Gregori [1], que concebe um cérebro de estrutura triádica. Se a aprendizagem é um processo de adaptação ao meio em constante transformação e os estilos de aprendizagem são, entre outras coisas, a maneira característica que os estudantes têm para se adaptar e viver o contexto, então, a forma como utilizarem suas funções cerebrais definirá seu estilo de pensamento e aprendizagem. A pesquisa pretende levar aos estudantes de Engenharia de Software o desenvolvimento de seu potencial cerebral encaminhado a uma sólida formação integral que possibilite sua acertada inserção na vida laboral.

Palavras-chave: cérebro triádico, competências lógicas, criativas e operativas, currículo, engenharia de software. 


\section{Introducción}

En la actualidad, todas las áreas del conocimiento tienen alguna relación con los sistemas de información, concretamente con la Ingeniería de Software, la cual puede tomarse como parte integradora de cada una de las ramas de las ciencias. Las ingenierías manejan una serie de sistemas que deben ser estudiados para poder trabajar sobre ellos: es allí donde la Ingeniería de Software (IS), con su análisis, diseño, modelación y desarrollo de los sistemas, se constituye en forma de soporte eficiente.

Lo anterior explica el porqué del acelerado crecimiento y necesidad de ingenieros en el área de las TIC en el país, lo cual ha llevado a que el número de programas relacionados vaya en aumento vertiginoso en los últimos años.

De acuerdo con el documento de Acis (Asociación Colombiana de Ingenieros de Sistemas) [2], la orientación que se les ha dado a los programas de Ingeniería de Sistemas en Colombia, ofrecidos por las diferentes instituciones de educación superior, y los porcentajes de los programas con cada orientación, se distribuye como se observa en la tabla 1:

Tabla 1. Áreas caracterización del conocimiento

\begin{tabular}{|l|c|}
\hline \multicolumn{1}{|c|}{ Descripción } & Porcentaje \\
\hline Ciencia de la computación & $17 \%$ \\
\hline Ingeniería de software & $39 \%$ \\
\hline Sistemas de información & $12 \%$ \\
\hline Tecnología de la información & $5 \%$ \\
\hline Otros & $27 \%$ \\
\hline
\end{tabular}

Fuente: ACIS [2]

En lo que respecta a las universidades colombianas, el reto que tienen en la actualidad es interpretar los procesos sociales orbitales y los problemas que obstaculizan la construcción de un país más justo, equitativo e innovador.

Salvia [3] afirma que el profesional debe recibir una educación integral. Por ello la formación del ingeniero de software colombiano debe suponer el desarrollo de competencias intelectuales básicas para la sociedad del conocimiento, en la cual se verá inmerso, habilidades específicas en tecnología y competencias comunicativas que dinamicen su creatividad para desarrollar soluciones que den respuesta idónea a fenómenos que afectan al mundo y a su entorno. Lo anterior puede lograrse aplicando las herramientas fundamentales de la educación para la convivencia con miras al cambio social: "la proyección social, el trabajo en equipo y el liderazgo creativo" [4, p. 2].
Según el proyecto swEвоK (Software Engineering Body of Knowledge) que constituye una guía elaborada por el IEEE Cs [5], la cual recoge los contenidos del conocimiento de ingeniería del software, documento que actualmente es un informe técnico de ISO E IEC con la referencia ISO/IEC TR, "la tendencia de los profesionales de ingeniería en el ámbito mundial se enfoca a la formulación de guías generales para análisis, diseño, desarrollo y a la adaptación de software acorde con los diversos proyectos organizacionales, teniendo en cuenta parámetros de conocimiento". La tendencia en lo concerniente al proceso de desarrollo, utiliza metodologías genéricas, como por ejemplo, el Unified Process, dejando a un lado aquellos esquemas rígidos, de los años ochenta.

En la educación tecnológica en el área de sistemas, se resalta el propósito de formar competencias en el desarrollo de software, bajo criterios de efectividad en la comunicación entre el cliente y el usuario para lograr un producto que satisfaga las necesidades de las empresas.

De otra parte se debe tener presente la práctica, la cual, según afirma Zabalza [6], sirve para: aproximar a los estudiantes a escenarios profesionales reales; ir organizando y reorganizando marcos de referencia que les sirvan para entender mejor los conceptos y contenidos estudiados en la carrera; detectar los puntos fuertes y débiles de cada uno; reconocer las propias aptitudes, actitudes y competencias personales - sobre todo las referentes a las relaciones interpersonales-; detectar las lagunas en la propia preparación y reconocer las necesidades de formación y reflexionar sobre lo que cada uno ha hecho y aprendido durante el período de prácticas revisando conscientemente la experiencia vivida. Las prácticas conducen en muchos casos a que los estudiantes obtengan su primer empleo.

En cuanto a la perspectiva de la especialización, esta ha decaído y lo que predomina es el trabajo en equipo y los profesionales multifuncionales. Esto significa el desarrollo de una nueva habilidad, denominada por Aguirre y Calama [7] como la de sintetizar la complejidad, es decir, capacidades que sean congruentes con la estrategia de este trabajo multiprofesional.

El avance acelerado de las tecnologías de la información y de las comunicaciones está impactando fundamentalmente en dos niveles, según Maté [8]; por un lado, agiliza la transferencia de información entre organizaciones, y, por otro, está produciendo paulatinamente un proceso de democratización de la accesibilidad a la información, lo cual conlleva cambios profundos en las organizaciones. Esta rápida evolución del conocimiento, y su facilidad de acceso, hace que la formación inicial de los 
estudiantes deba enfocarse al desarrollo de capacidades intelectuales de percepción y comprensión, no sólo como parte de su capacitación para el primer empleo, sino como base para la mejora de su nivel de competencia y los posibles cambios de orientación a lo largo de su vida activa.

Para lo anterior, Montes [9] propone cuatro objetivos generales:

a) desarrollar el deseo de aprender y los hábitos de búsqueda del conocimiento en fuentes diversas; b) enfrentarse con problemas nuevos, plantearlos adecuadamente, analizarlos sistemáticamente y lograr soluciones; c) investigar las relaciones funcionales entre lo que se aprende y el mundo real, incluyendo el ámbito laboral y las relaciones humanas; d) desarrollar una visión integradora, que posibilite el análisis de las dimensiones de cualquier problema o situación de la vida real, sin basarse exclusivamente en la percepción parcial aportada por una especialidad académica concreta [9, p. 24].

En consecuencia, y según se ha avanzado en el presente milenio, un elemento esencial en la educación en ingeniería es la formación humana a partir del diagnóstico de necesidades sociales, donde desarrollar calidad humana debería ser previo a desarrollar calidad profesional, por cuanto es lo que redunda en un alto impacto social.

Con la globalización de la industria y el mercado laboral han aparecido nuevas doctrinas profesionales que siguen tendencias como, según Espriu [10]: economía global, interdependencia, transnacionalización empresarial; el inglés como idioma de negocios universal; la era de la información y sus consecuencias - la venta de conocimientos como área prioritaria-; normalización internacional de productos, normas de calidad internacional; cambio de valores, tanto en la empresa como en los individuos; tecnología educativa - televisión, comunicaciones, medios multimedia, etcétera-; nuevas redes internacionales; posibilidades multimedia y el CD-ROM con su enorme potencial; múltiple interacción entre las universidades y una creciente importancia de la transferencia de tecnología.

Pero lo que se observa es que las universidades continúan desarrollando currículos monádicos centrados en el desarrollo de la lógica matemática, cuando estos se deben reestructurar para que se despliegue en los estudiantes un pensamiento crítico, que les permita madurar las competencias del saber, hacer y del ser, desde un enfoque tricerebral para construir propuestas contextualizadas que den respuesta a las necesidades del sector productivo.
Es así que desde la cibernética social se entiende el currículo como un conjunto de agendas formales e informales para capacitar el cerebro unitríadico de las personas en la autoconducción, el autosostenimiento y la autoeducación, de manera que pueda integrarse socialmente como protagonista en el juego tríadico proporcional de la vida. El currículo tiene cuatro niveles de actuación en sus funciones mentales, que van de lo más simple a lo más complejo, nivel de ejecución, de conducción, de asesoría y de liderazgo. Reconoce por lo menos tres funciones: lógicas, operativas y emocionales. La clave es formar personas integras pero proporcionales, potenciando los talentos y habilidades.

$\mathrm{Y}$ en cuanto a investigación sobre el tema no se registra en Colombia ningún proyecto realizado en universidad alguna, en razón a que la ingeniería de software como pregrado es un campo novedoso en el país y ante el Ministerio de Educación. Por este motivo, se hace referencia a las agremiaciones como ACIS, SWEBOK, IEEE, que son las entidades que prestan su concurso en la regulación del mismo.

En lo que respecta a la Fundación Universitaria Inpahu, el programa de Ingeniería de Software nació motivado por las inquietudes de los estudiantes de Tecnología en Sistemas de continuar sus estudios profesionales, y fue diseñado bajo la estructura del modelo curricular con base en competencias profesionales, formando parte de los Programas de la Facultad de Nuevas Tecnologías.

En una investigación de mercados contratada por Inpahu en el 2009, y adelantada por la empresa Conocer y Proyectar [11] con el fin de identificar las necesidades y expectativas empresariales, en la cual se consultaron empresas como Quala, Avantel, Ministerio de Hacienda, Banco Santander, McDonald's, Universidad de los Andes, Alcatel, se destaca a manera de conclusión que en el perfil ideal de los egresados de carreras técnicas y tecnológicas no señalan una preparación académica en aspectos como: tolerancia, solución de problemas, trabajo en equipo y capacidad de adaptación y sostenimiento en grupos de trabajo.

A lo anterior se suma el hecho de haber observado en los estudiantes de Tecnología de Sistemas, actualmente egresados, dificultades en comunicación oral y escrita, establecimiento de relaciones, argumentación sustentada, proposición de alternativas, soluciones y construcción de un pensamiento propio. Del mismo modo, se advirtió un desarrollo de pensamiento práctico limitado a cumplir con acciones básicas del proceso enseñanza-aprendizaje, tales como desarrollar tareas, contestar cuestionarios, entre otras, sin avanzar hacia la planeación y liderazgo propositivo. 
Así mismo, a lo largo del proceso educativo se mantuvieron bajos niveles de atención y concentración, falta de disciplina de estudio, dificultades para el cumplimiento de las normas, poca responsabilidad y sentido de pertenencia, poco desarrollo de liderazgo y trabajo en grupo. Esto condujo a reflexionar sobre el perfil que se espera del futuro ingeniero de software egresado de la Fundación Universitaria Inpahu, consultando con el que se esperaba al momento de la concepción del modelo curricular, suscitando la necesidad de un ajuste en cuanto a que debería mostrar básicamente una proyección de competencias intelectuales articuladas con las competencias socioemocionales, integrando los aspectos cognitivos, emocional y procedimental en los contenidos programáticos. Al respecto, Velandia [12] manifiesta que:

[...] la educación de los sentimientos no ha sido tema preferente en la formación universitaria. Nos hemos centrado en las competencias intelectuales dejando en un segundo plano la educación de las emociones, la afectividad y los sentimientos, dando lugar a una desproporcionalidad entre lo cognitivo, lo emocional y procedimental en los contenidos curriculares [12, p. 1].

Si Inpahu quiere formar un ingeniero de software competitivo en el mercado actual, debería ser un profesional integral y en consecuencia los currículos deberían adecuarse para responder debidamente a las nuevas situaciones.

Así las cosas, se definió como objetivo de la investigación la reestructuración de la malla curricular del programa de Ingeniería de Software de la Fundación Universitaria Inpahu, con la implementación de una propuesta pedagógica basada en el desarrollo de las competencias lógicas, creativas y operativas, tomando como hipótesis el que la metodología utilizada por los docentes no desarrolla las competencias lógicas, operativas y creativas en forma integral.

\section{Método}

A partir del hológrafo social de Velandia [13] se define el Ciclo Cibernético de Transformación сст como un proceso de organización de acción humana que integra tres fases fundamentales: 1. Proceso lógico-analítico o fase de investigación, 2. Proceso intuitivo-sintético o fase de propuesta y 3. Proceso motriz operacional o fase tecnológica.

En la primera fase, se parte de un tema o problema, se realiza la colecta de datos, el procesamiento, y se con- cluye con el diagnóstico. En la segunda fase, se parte de dicho diagnóstico para construir una propuesta de intervención a nivel correctivo o de mejoramiento, pasando por los pasos de exploración de alternativas (futurización y creatividad), definición de metas y programación para la construcción de la propuesta. En la tercera fase se implementa la propuesta diseñada en la fase anterior, a través de los pasos de implantación, acompañamiento y retroalimentación o feedback, que dan origen a una nueva realidad y otra posibilidad secuencial de continuar con el ciclo de transformación.

La diferencia con el método científico tradicional se encuentra en que bajo la propuesta del CCT el proceso investigativo no culmina en el diagnóstico sino que se operacionaliza en una propuesta que debe ser ejecutada, evaluada y redireccionada.

La metodología utilizada es mixta (descriptiva-cuantitativa), por cuanto se centra en caracterizar la realidad educativa investigada y está basada en el diseño explicativo secuencial Dexplis [14]. El proyecto se caracteriza por el análisis de datos cuantitativos en su primera fase. En una segunda fase, se recogen y evalúan datos cualitativos. Este proceso híbrido se concibe cuando los resultados cuantitativos iniciales informan la recolección de los datos cualitativos. Cabe precisar que la segunda fase se construye con los resultados de la primera. Finalmente, los resultados de las dos fases se integran para la interpretación y elaboración del reporte de estudio.

\subsection{Población}

El proyecto se desarrolló con una población de 136 egresados pertenecientes a la carrera de Tecnología en Sistemas en la Fundación Universitaria Inpahu, y en la Fundación Universitaria CIDCA, y 20 decanos y directores de carrera de dichas instituciones. Se consultaron egresados de CIDCA, básicamente para contar con información de otra institución y por la facilidad de acceder a ella.

\subsubsection{Proceso recolección de información}

A 80 estudiantes de Inpahu y 20 de CIDCA se les realizó una encuesta diseñada con diez preguntas cerradas y organizadas por bloques cuyo contenido tuvo como fuente la actual malla curricular existente para la carrera de ingeniería de software en la Fundación Universitaria Inpahu (ver anexo 1). El primer bloque de preguntas hace relación al área de ingeniería de software dentro de la ingeniería de sistemas; el segundo, se refiere al desarrollo de las competencias socioemocionales en el futuro ingeniero de software; el tercero, al desarrollo de las competencias 
intelectuales en el estudiante; el cuarto indaga por el desarrollo de las competencias procedimentales; y el quinto tiene que ver con la sensibilización y la importancia de la temática en la población.

Así mismo, se realizó una entrevista a los 20 decanos o directores de carrera de Ingeniería de Sistemas, en las cuales se consideran aspectos relevantes como la problemática, las causas, conocimientos relacionados con el tema e importancia de la reestructuración de la malla curricular para la formación del futuro ingeniero de software.

Por último, se implementó el Revelador del cociente mental triádico de De Gregori [1], como una herramienta para medir el potencial mental de los estudiantes y corroborar si los futuros ingenieros tenían más desarrollado su cerebro izquierdo.

\subsubsection{Selección de la muestra}

Se determinó teniendo en cuenta el mercado objetivo, el cual estuvo conformado por tecnólogos egresados de las universidades Fundación Universitaria Inpahu y la Fundación CIDCA durante los últimos cuatro semestres, que corresponde a los años 2009 y 2010 , la cual está conformada por una población de 136 exalumnos, según fuentes estadísticas de las Oficina de Registro y Control de las universidades en mención. Un nivel de confianza de $95 \%$ y un grado de error de $5 \%$ en la fórmula de la muestra.

Nivel de confianza $(Z)=1,96(95 \%)$

Grado de error $(\mathrm{e})=0,05(5 \%)$

Universo $(\mathrm{N})=136$

Probabilidad de ocurrencia $(\mathrm{P})=0,5$

Probabilidad de no ocurrencia $(\mathrm{Q})=0,5$

$$
\begin{aligned}
\mathrm{n}= & \left(\mathrm{Z}^{2} \cdot \mathrm{P} \cdot \mathrm{Q} \cdot \mathrm{N}\right) /\left(\mathrm{N} \cdot \mathrm{e}^{2}+\mathrm{Z}^{2} \cdot 0,5 \cdot 0,5\right) \\
\mathrm{n}= & \left((196)^{2}(0,5)(0,5)(136)\right) /\left((136)(0,05)^{2}+\right. \\
& \left.(196)^{2}(0,5)(0,5)\right) \\
\mathrm{n}= & (((3,84)(0,25)(136)) /((136)(0,0025)+(3,84) \\
& (0,25)) \\
\mathrm{n}= & 130,6 / 0,34+0,96 \\
\mathrm{n}= & 130,6 / 1,3 \\
\mathrm{n}= & 100,3
\end{aligned}
$$

De acuerdo con la anterior fórmula de Morales [15], la muestra corresponde a 100 tecnólogos exalumnos de las universidades Inpahu y CIDCA.

\section{Resultados}

\subsection{Procesamiento de la información}

Se dividió en tres momentos: 1 . datos de encuestas, 2. datos de la entrevista y 3 . datos del RCMT.

\subsubsection{Datos de encuesta a egresados de Tecnología en Sistemas}

De los resultados obtenidos, el total de encuestados está de acuerdo con que exista una ingeniería de software que desarrolle en el futuro profesional las competencias lógicas, operativas y afectivas. Un 70\% manifiesta la falta de contenidos programáticos orientados hacia el desarrollo de competencias emocionales en los tecnólogos en sistemas. Respecto a las asignaturas que consideran deben hacer parte de la malla curricular para lograr dicho desarrollo, mencionan el cerebro y su aplicación a la ingeniería como la más necesaria, seguida por liderazgo transformacional (figura 1).

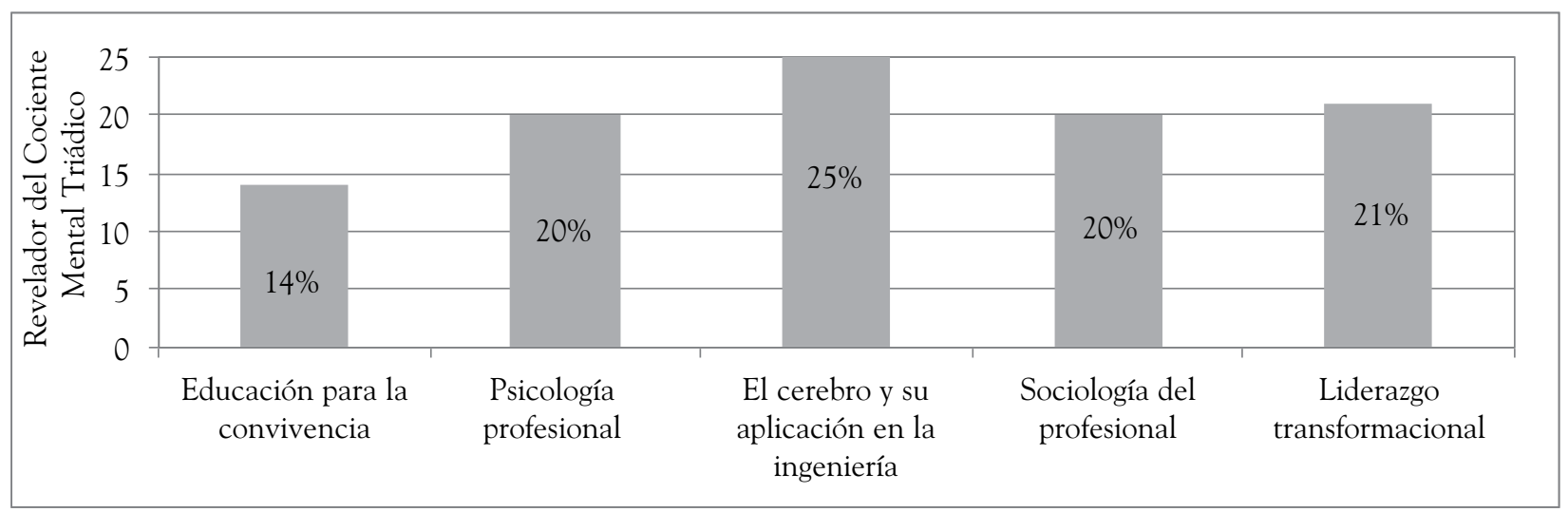

Figura 1. Asignaturas para el desarrollo de competencias socioemocionales Fuente: los autores 
En cuanto al desarrollo de las competencias lógicas, el $70 \%$ de los encuestados señala que los contenidos programáticos son suficientes. No obstante, manifiestan que se debe reforzar básicamente en desarrollo de software con tecnología de punta y lenguajes de programación (figura 2).

Para el desarrollo de competencias de negocios en el futuro ingeniero de software, los egresados indican respecto de las asignaturas suministradas, en primer lugar, la Ingeniería de Negocios, seguida por Matemática financiera y Macroeconomía como las áreas fundamentales en el manejo de la economía y los negocios de la ingeniería. Esto demuestra su interés por relacionar la ingeniería del software con los mercados (figura 3).

\subsubsection{Datos de la entrevista a decanos y directores de carrera}

Entre los resultados por resaltar frente al desarrollo de las competencias intelectuales (lógicas) en el futuro ingeniero de software, concretamente qué aspectos deberían cumplir las áreas del conocimiento para reforzar en la nueva malla curricular para el profesional de esta carrera, se encontró que todos los entrevistados manifiestan la necesidad de ajustar la malla curricular de acuerdo con las necesidades del mercado y, por lo tanto, estos ajustes deben ser constantes. De igual manera, respecto al desarrollo de las competencias procedimentales (operativas), qué temas deberían cubrir las áreas del conocimiento al reestructurar una malla curricular para el nuevo profesional de esta carrera, la mayoría de respuestas se dieron en la necesidad de que el ingeniero de software aprenda a vender su imagen y sus productos. Por último, en cuanto al desarrollo de competencias socioemocionales, todos estuvieron de acuerdo en la necesidad de trabajar las competencias creativas.

\subsubsection{Datos del RCMT}

Test aplicado a 30 egresados entre hombres y mujeres seleccionados al azar. Evaluando promedios, se

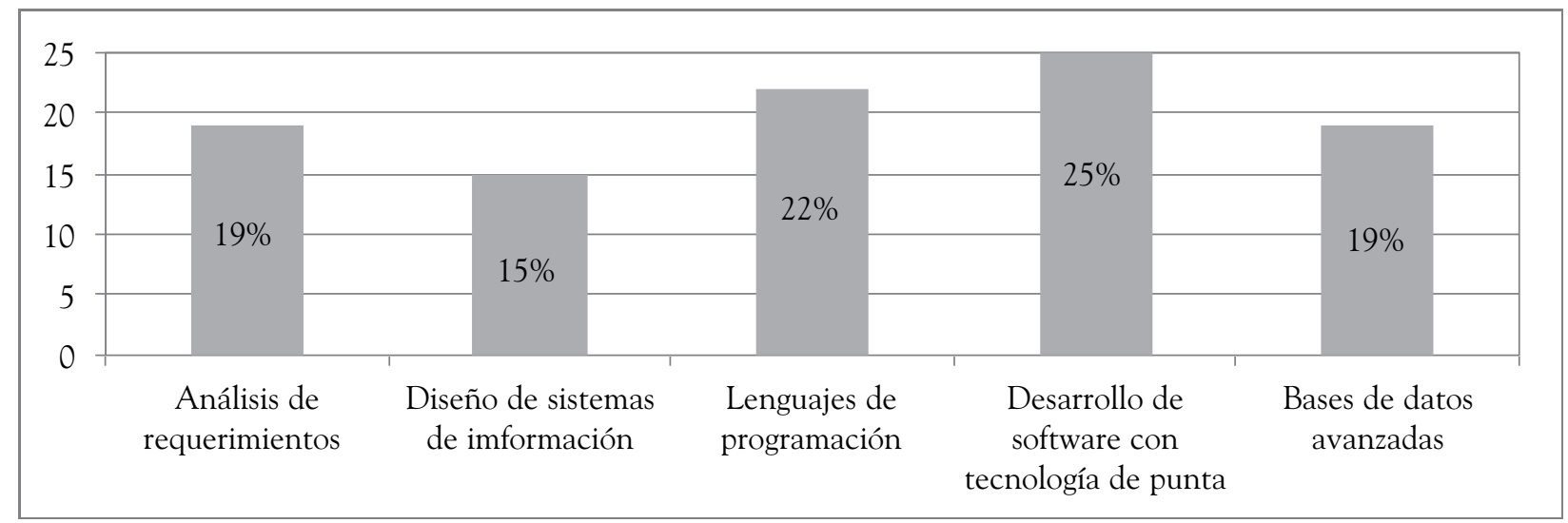

Figura 2. Asignaturas para el desarrollo de competencias intelectuales

Fuente: los autores

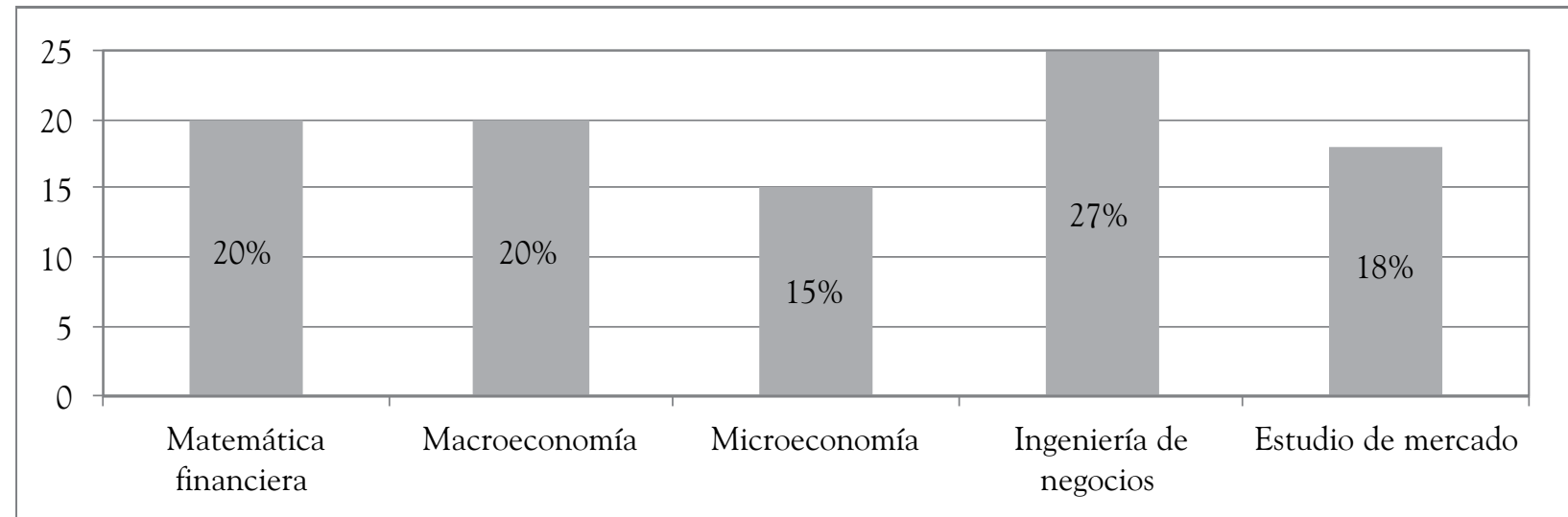

Figura 3. Competencias de negocios

Fuente: los autores 
observa como dominante el cerebro derecho o creativo con un 35\%; en un segundo lugar, y muy de cerca, se encuentra el cerebro central con un $34 \%$, y en un $31 \%$ del promedio de estudiantes predomina el cerebro izquierdo o lógico (figura 4).

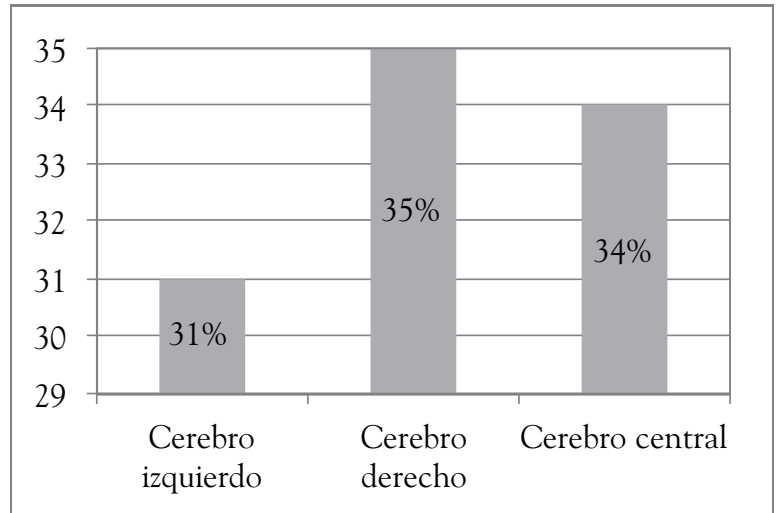

Figura 4. Cerebro dominante de los egresados de Tecnología en Sistemas

Fuente: los autores

\section{Discusión y conclusiones}

Contrario a lo esperado, es decir, que predominara la competencia lógica en los egresados de Tecnología de Sistemas, y futuros Ingenieros de Software, se observa que tienen más desarrolladas las capacidades creativas. Esta característica favorece la implementación de un nuevo plan curricular orientado al desarrollo de competencias por los tres cerebros, buscando incentivar el cerebro lógico y aprovechando las competencias creativas para un mejor aprendizaje, por cuanto el cerebro derecho desarrolla la capacidad creativa, emocional, mística, ética y estética. Esto afianza la creatividad e innovación tecnológica, el sentido de pertenencia y las relaciones interpersonales. En la Fundación Universitaria Inpahu se debe asegurar la pertinencia del currículo y establecer los mecanismos de adaptación a los cambios que se requieren para que el futuro ingeniero madure las competencias socioemocionales indispensables para ello.

Es igualmente importante favorecer la vocación del estudiante, enriquecer las opciones de estudio, de investigación y de ejercicio profesional: de esa manera los egresados en su conjunto pueden brindar amplias y variadas respuestas a las demandas de la sociedad. Esta flexibilidad ayuda a que el estudiante se motive y se comprometa con el aprendizaje, más allá del cumplimiento con las exigencias de un plan de estudio.
Por otra parte, los currículos actuales han definido las alternativas de formación de los ingenieros únicamente dentro del alcance de las áreas del ciclo profesional del programa. Sin embargo, los cambios continuos a los cuales están sometidas las organizaciones y la diversidad de alternativas tecnológicas que surgen día a día demandan perfiles particulares de formación, dentro de cada rama de la ingeniería, que no están al alcance de las áreas del ciclo profesional definidas en el momento de la concepción del modelo curricular. Esto provoca insatisfacción en las organizaciones que contratan los egresados del programa, pues deben invertir en formación adicional de sus empleados.

En este sentido, la Facultad de Ingeniería y Tecnologías de la Información de la Fundación Universitaria Inpahu debe prestar atención especial a los problemas y necesidades sociales que atiende actualmente la ingeniería nacional, para traducirlos en unos propósitos de formación del Ingeniero de Software del futuro, como profesional, pero también como hombre y ciudadano.

En un currículo manejado por proyectos, la clase es uno dentro de muchos métodos o técnicas que existen; no se plantea que tenga que desaparecer, cada cual la utilizará cuando lo considere conveniente. Sin embargo, hay que tener una mirada abierta hacia las posibilidades didácticas. Debe pensarse que las soluciones a los problemas que se derivan de los proyectos pueden encontrarse en una clase, o en una biblioteca, en una oficina, en una charla en la cafetería, en una llamada telefónica, en una conferencia televisada, en una consulta a través de Internet $\mathrm{o}$ a otro profesor, $\mathrm{o}$ a otra biblioteca, etcétera.

Los cambios sociales, así como el desarrollo científico y tecnológico, exigen que los currículos deban adaptarse permanentemente para responder adecuadamente. En un currículo centrado en proyectos, estos demandan la introducción de nuevos saberes y de nuevos recursos, así como la adaptación de sus objetivos y sus contenidos con el fin de satisfacer las necesidades de formación. Se evidencia, por tanto, en Inpahu, la necesidad de implementar una malla curricular integral para que no sólo se desarrollen competencias lógicas, sino también operativas y creativas.

Por lo anterior, se plantea la construcción de los lineamientos de una propuesta pedagógica alternativa apoyada en la Cibernética Social, para la reestructuración e implementación de un currículo que potencie el desarrollo de las competencias lógicas, operativas y creativas en la Fundación Universitaria Inpahu, y que responda a las necesidades, perfiles y desempeños que requieren las empresas de Ingenieros de Software, respetando las directrices del Ministerio de Educación Nacional en el Decreto 2145 de 
2010 y las políticas institucionales, de tal forma que se dé respuesta a los perfiles que exige el sector productivo.

A través del currículo, se debe desarrollar en los estudiantes la capacidad de seguir aprendiendo. La ciencia se encuentra en permanente construcción, por lo cual hay que ofrecer a cada estudiante las herramientas conceptuales y metodológicas necesarias, no sólo para acceder a los conocimientos básicos, sino para la formación y desarrollo de su capacidad crítica, a fin de construir una sociedad avanzada tecnológicamente, pero a la vez humana, solidaria y comprometida.

\subsection{Propuesta}

La propuesta que se diseñó para la transformación curricular del Programa Ingeniería de Software de la Fundación Universitaria Inpahu se realizó con el fin de potenciar las competencias lógicas, creativas y operativas desde un enfoque tricerebral apoyado en la Cibernética Social. Con esta propuesta también se busca acoplar elementos que el profesional debe brindarle a la sociedad como son: investigación, servicios a la sociedad, formación integral y pensamiento crítico, de tal forma que el estudiante se vincule a proyectos que impulsen el desarrollo social, económico y cultural del país, para que así pueda intervenir con solvencia científica, tecnológica, ética y buenas relaciones sociohumanas y culturales centradas en el dominio del conocimiento, de la información y de la computación en el contexto nacional e internacional.

Lo anterior va en sintonía con el PEI de Inpahu, que presenta un modelo curricular por competencias, el cual concibe cuatro competencias básicas: complementaria, relacionada con la capacidad que permite al estudiante reconocer su situación académica, afectiva y ciudadana; disciplinar, se refiere a la capacidad que tiene el individuo de construir la praxis alrededor de comprender y tratar de manera holística y totalizadora una situación problémica; práctica-profesional, dirigida hacia la capacidad para actuar crítica, creativa y efectivamente en diversos escenarios de su desempeño profesional; e investigativa, entendida como la capacidad que tiene el sujeto de indagar e interrogarse de manera permanente sobre el objeto de su disciplina, su profesión, su sentido epistemológico y su ser, en pos de proponer nuevas alternativas de desarrollo y comprensión de las mismas.

La matriz curricular que se propone está basada en la matriz triple escalera de la vida de Gregori. La construcción contempla tres dimensiones, cada una con cuatro niveles de conocimiento, como se ve a continuación.
La dimensión lógica, referente a fundamentación de teorías, hechos y conceptos, en la que el cuarto nivel desarrolla una competencia propositiva que permite sustentar todos los argumentos desde un enfoque tecnológico y científico en función de la solución de problemas informáticos, argumentando las respuestas desde una posición crítica. El tercero se refiere a una competencia investigativa que busca desplegar propuestas y soluciones con criterios de paradigmas y modelos que suministra la ciencia. En el segundo nivel se utilizan técnicas de recolección y clasificación de datos para la aplicación de técnicas de ingeniería de software en la aplicación de soluciones informáticas. Finalmente, en el primer nivel se desarrolla una competencia comunicativa para socializar de forma oral y escrita los avances y resultados de las metodologías de aprendizaje.

En la dimensión operativa propia de aplicaciones, prácticas, ejecuciones con eficacia, el cuarto nivel corresponde a la competencia administrativa que propicie la formación de profesionales con propósitos de gestión, administración e innovación de proyectos informáticos que eleven su capacidad profesional en el sector productivo con liderazgo y apropiación del conocimiento tecnológico. En el tercer nivel hay una competencia de planeación para organizar todos los procesos de financiación de proyectos, licitaciones, ventas y gestión de proyectos informáticos. En el segundo, se realiza una competencia profesional que permita utilizar herramientas conceptuales, metodológicas y técnicas para la elaboración de las soluciones informáticas con calidad. Por último, en el primer nivel se busca desarrollar una competencia valorativa, que aprecia la versatilidad de las herramientas y metodologías para enriquecer el desempeño profesional combinado con la tecnología.

La dimensión emocional caracterizada por un ambiente lúdico-estético trascendente que permite desarrollar el juego, la convivencia la creatividad y la estética y su nivel de conocimiento en el individuo, propone en su cuarto nivel una competencia estética para demostrar apropiación del conocimiento a través de propuestas pedagógicas lúdicas y de trascendencia social. En el tercer nivel se desarrolla una competencia proyectiva hacia escenarios favorables con las aplicaciones de las nuevas metodologías tecnológicas y gestión del conocimiento adquirido durante la carrera. En el segundo nivel se propone una competencia creativa con el fin de abrir nuevas posibilidades para el análisis, diseño y desarrollo de aplicaciones que den solución a los problemas informáticos, generando productos con buena estética óptica al usuario final. En el primer nivel se realiza una competencia afectiva que busca construir re- 
laciones afectivas a través del trabajo en equipos y el compartir saberes, para el mejoramiento de la calidad de vida del profesional, de tal forma que sea sensible a la naturale$\mathrm{za}$, a los seres humanos y a todo el entorno ecosistémico.
Teniendo en cuenta lo expuesto, se presenta el diseño de la matriz curricular para la Ingeniería de software de Inpahu, diseñada según las tres funciones tricerebrales y sus niveles de actuación (tabla 2).

Tabla 2. Matriz curricular aplicada a estudiantes de Ingeniería de Software

\begin{tabular}{|c|c|c|}
\hline PENSAR-SABER & DISCUTIR-HACER & SENTIR-SER \\
\hline \multicolumn{3}{|c|}{ Niveles Tricerebrales de Actuación del Profesional en Ingeniería de Software } \\
\hline Epistemología & Administración & Ludomistetica \\
\hline $\begin{array}{l}\text { El estudiante tiene habilidades de pensamiento } \\
\text { crítico, que le permiten argumentar y proponer, } \\
\text { debido a que posee técnicas de recolección } \\
\text { de información que le posibilita adquirir un } \\
\text { pensamiento propio. }\end{array}$ & $\begin{array}{l}\text { El estudiante está en capacidad de realizar } \\
\text { actividades soporte técnico, digitación y } \\
\text { análisis. }\end{array}$ & $\begin{array}{l}\text { El estudiante es ético, con conocimientos de } \\
\text { lectura y escritura. }\end{array}$ \\
\hline Investigación & Planeación & Nivel-Alfa \\
\hline $\begin{array}{l}\text { El estudiante desarrolla una actitud científica, con } \\
\text { dominio del lenguaje técnico utilizado en el argot } \\
\text { computacional. }\end{array}$ & $\begin{array}{l}\text { El estudiante aplica los conocimientos de } \\
\text { lenguajes de programación con ayuda de } \\
\text { los docentes y tutores. }\end{array}$ & $\begin{array}{l}\text { El estudiante futuriza sus conocimientos } \\
\text { en el ambiente laboral donde va ejercer. } \\
\text { (Entidades gubernamentales, entidades } \\
\text { privadas, empresarios, etc.). }\end{array}$ \\
\hline Clasificación & Profesión & Creatividad \\
\hline $\begin{array}{l}\text { El estudiante domina y clasifica las fuentes de } \\
\text { información recibidas en el entorno computacional e } \\
\text { informático. }\end{array}$ & $\begin{array}{l}\text { El estudiante adopta sus conocimientos } \\
\text { teóricos, técnicos científicos al campo de } \\
\text { la computación. }\end{array}$ & $\begin{array}{l}\text { El estudiante tiene un desarrollo creativo } \\
\text { respecto a las prácticas y creación y diseño } \\
\text { de software. }\end{array}$ \\
\hline Comunicación & Sobrevivencia & Sensibilidad \\
\hline $\begin{array}{l}\text { El estudiante expresa cotidianidad en la lógica, } \\
\text { posee destreza en lenguaje natural y desarrollo de } \\
\text { algoritmos. }\end{array}$ & $\begin{array}{l}\text { El estudiante tiene interrelación con } \\
\text { equipos de cómputo conoce la esencia de } \\
\text { la teoría de los sistemas. }\end{array}$ & El estudiante es sensible al trabajo en grupo. \\
\hline
\end{tabular}

Fuente: elaboración propia

La propuesta curricular está ligada a la potenciación de las competencias del ser, saber y saber hacer a través de las herramientas de la cibernética social a nivel personal $\mathrm{y}$ de equipo en los estudiantes, y se direcciona a favorecer el progreso de habilidades con enfoque tríadico, que se traduzcan en el aula en acciones de enseñanza-aprendizaje para el equilibrio y la proporcionalidad en el desarrollo del cerebro de los estudiantes.

\section{Referencias}

[1] W. de Gregori, Construcción Familiar y Escolar de los 3 Cerebros, Bogotá: Editorial Kimpres, 2002, pp. 34-37.

[2] ACIs, Caracterización de la ingeniería de sistemas y programas afines en Colombia, Bogotá: Asociación Colombiana de Ingenieros de Sistemas, 2006.

[3] A. Salvia. "Crisis del empleo y nueva marginalidad en tiempos de cambio social. Génesis de una catástrofe anunciada”, en Revista Instituto de estudios y formación central de los trabajadores. Argumentos, No. 4, IIfCs,
Instituto de Investigaciones Gino Germani, Facultad de Ciencias Sociales, UBA, Buenos Aires, 2004.

[4] M. A. Velandia. Estrategias para construir la convivencia solidaria en el aula universitaria, Bogotá: Editorial Universidad Cooperativa de Colombia, 2006.

[5] IEeE CS. Proyecto SWeboK. ISo/IEC TR, 2011. [En línea]. Disponible en: http://www.idg.es/computerworld/articulo.asp?id=

[6] M. A. Zabalza, "El Prácticum y los Centros de Desarrollo Profesional", en AA.VV., Evaluación de experiencias $y$ tendencias en la formación del profesorado, Bilbao: ICEUniversidad Deusto, 1996, p. 266.

[7] G. Aguirre y J. M. Calama, "Una nueva enseñanza universitaria para las profesiones técnicas", En II Jornadas Nacionales de Innovación en las Enseñanzas de las Ingenierías. Comunicaciones. Vol. 2, ICE, Universidad Politécnica de Madrid, pp. 1101-1105, 1997.

[8] J. L. Maté Hernández, "Utilización de las nuevas tecnologías en la formación”, en I Encuentro Iberoamericano de Directivos en las Enseñanzas de Ingeniería, Ponencias, ICE de la Universidad Politécnica de Madrid, pp. 119-127, 1999. 
[9] J. M. Montes Villalón, “Tecnología y educación”, en Aparicio y González, Tecnología y sociedad, ICE-Universidad Politécnica de Madrid, pp. 124-153, 1991.

[10] J. J. Espriu. Continuous Education for Engineers in Front of New Challenges and Old Problems. Proceedings of World Congress of Engineering Educators and Industry Leaders, Vol. 1, París: Unesco, pp. 21-26, Jul., 1996.

[11] Conocer y Proyectar. Informe de Investigación de Mercados. Necesidades y expectativas de Empresarios. Bogotá, Sep., 2009.

[12] M. A. Velandia. Competencias sociemocionales. Alicante (España). Universidad Cooperativa de Colombia, Maes- tría en Educación, Módulo de Educación para la Convivencia, 2011. [En línea]. Disponible en: http://es.scribd. com/doc/55938904/Competencias-Socioemocionales

[13] C. Velandia. Metodología interdisciplinaria centrada en equipos de aprendizaje MICEA, Bogotá; Universidad Cooperativa de Colombia, 2005.

[14] R. Hernández, C. Fernández, y P. Baptista, Metodología de la investigación, 5a Ed., México: McGraw-Hill, 2010.

[15] P. Morales, Estadística aplicada a las ciencias sociales. Tamaño necesario de la muestra, Dic., 2012. [En línea]. Disponible en: http://www.upcomillas.es/personal/peter/investigacion/Tama\%F1oMuestra.pdf

\section{Anexo 1}

\section{Instrumento}

\section{Estimado(a) Señor(a):}

Reciba un cordial saludo. Estamos desarrollando una investigación de los planes de estudio en los programas de Ingeniería de Sistemas y programas afines con Ingeniería de Software. Para nosotros es muy importante contar con sus apreciaciones sobre el tema. Agradecemos su colaboración al contestar todas las preguntas con claridad y objetividad para que el proceso tenga trascendencia en la formación de los futuros ingenieros de este país.

\section{Unidades de análisis}

\section{Datos generales.}

Nombre y apellidos:

Institución:

Dirección:

Cargo que desempeña:

\section{Unidades de análisis.}

1. La institución educativa cuenta con carreras afines a Ingeniería de Software?

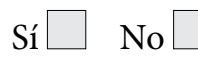

2. ¿En qué áreas de caracterización del conocimiento afines a la Ingeniería de Sistemas se destacan las carreras en su facultad?

Ingeniería de Software.

Mantenimiento de Computadores.

Sistemas Distribuidos.

Auditoria de Sistemas.

3. ¿ ¿ Usted considera que los contenidos programáticos de las materias que hacen parte del área de Ingeniería de Software desarrollan las competencias socioemocionales en el futuro profesional?

Sí $\square$ No $\square$ 
4. Teniendo en cuenta el desarrollo de las competencias socioemocionales en el futuro ingeniero de software, ¿cuál de las siguientes asignaturas considera usted que deben hacer parte de la malla curricular? Enumérelas en orden de importancia.

Educación para la convivencia

_ Psicología profesional

__ El cerebro en la ingeniería

__ Sociología del profesional

__ Liderazgo transformacional

5. ¿ ¿Usted considera que los contenidos programáticos de las materias que hacen parte del área de ingeniería de software son suficientes para desarrollar las competencias intelectuales en el futuro profesional?

Sí $\square$ No $\square$

6. En cuanto al desarrollo de las competencias intelectuales en el futuro ingeniero del software, y en orden de importancia, ¿en cuales áreas del conocimiento se debe reforzar la malla curricular para el nuevo profesional de esta carrera?

__ Análisis de requerimientos

_ Diseño de sistemas

__ Lenguajes de programación

___ Desarrollo con tecnología de punta

__ Bases de datos avanzadas

7. ¿ ¿Usted considera que los contenidos programáticos de las materias que hacen parte del área de Ingeniería de Software desarrollan las competencias procedímentales (saber hacer) en el futuro ingeniero con visión empresarial?

$$
\text { Sí } \square \text { No } \square
$$

8. Teniendo en cuenta el desarrollo de las competencias procedimentales y de negocios en el futuro ingeniero de software, ¿cuál de las siguientes asignaturas considera usted que deben hacer parte de la malla curricular? Enumérelas en orden de importancia.

___ Matemática Financiera

__ Macroeconomía

__ Microeconomía

__ Ingeniería de Negocios

Estudio de mercado.

9. ¿ ¿Usted considera que la Ingeniería de Sistemas en el área del desarrollo de software es competitiva en Colombia, con respecto a otros países de la región?

Sí $\square$ No $\square$

10. ¿Cómo considera la calidad del ingeniero dedicado al desarrollo de software en Colombia? Deficiente $\square \quad$ Regular $\square \quad$ Bueno $\square \quad$ Excelente $\square$ 